\title{
Evolution of zero-metallicity massive stars
}

\author{
Paola Marigo $^{1}$, Cesare Chiosi ${ }^{1}$, Léo Girardi ${ }^{2}$, and Rolf-Peter Kudritzki ${ }^{3}$ \\ ${ }^{1}$ Dipartimento di Astronomia, Università di Padova, \\ Vicolo dell'Osservatorio 2, I-35122, Padova, Italia \\ ${ }^{2}$ Osservatorio Astronomico di Trieste, \\ Via Tiepolo 11, I-34131, Trieste, Italia \\ ${ }^{3}$ Institute for Astronomy, University of Hawaii at Manoa, \\ 2680 Woodlawn Drive, Honolulu, HI 96822, USA
}

\begin{abstract}
We discuss the evolutionary properties of primordial massive and very massive stars, supposed to have formed from metal-free gas. Stellar models are presented over a large range of initial masses $\left(8 \mathrm{M}_{\odot} \lesssim M_{\mathrm{i}} \lesssim 1000 \mathrm{M}_{\odot}\right)$, covering the hydrogen- and helium-burning phases up to the onset of carbon burning. In most cases the evolution is followed at constant mass. To estimate the possible effect of mass loss via stellar winds, recent analytic formalisms for the mass-loss rates are applied to the very massive models $\left(M_{\mathrm{i}} \geq 120 \mathrm{M}_{\odot}\right)$.
\end{abstract}

\section{Introduction}

Over the years the existence of a primeval generation of (very) massive stars has been invoked in relation to various astrophysical issues (see Carr et al. 1984; Weiss et al. 2000 for extended reviews), such as: (i) fill the gap between the chemical abundances predicted by the Big Bang nucleosynthesis and those measured in Population-II stars; ( $i i)$ offer a viable solution to the G-dwarf problem in the solar neighbourhood; (iii) explain the observed enhancement in $\alpha$-elements observed in metal-poor stars, as well as the chemical abundances in the intergalactic medium as inferred from the spectra of high- $z$ damped Ly $\alpha$ systems; $(i v)$ contribute to the cosmological helium abundance; $(v)$ cause the re-ionization of the early Universe; and ( $v i$ ) provide a source of dark matter, in form of stellar remnants, as required for galactic haloes and galaxy clusters.

Several models of Population-III stars were constructed in the 1980-ies (e.g., El Eid et al. 1983; Ober et al. 1983). In recent years, the renewed interest from the cosmologic field has again spurred several studies on primordial stellar evolution (e.g., Marigo et al. 2001; Heger \& Woosley 2002).

\section{Evolutionary stellar models}

In this context we have carried out extensive evolutionary calculations, over a large range of stellar masses $\left(0.7 \mathrm{M}_{\odot} \lesssim M_{\mathrm{i}} \lesssim 1000 \mathrm{M}_{\odot}\right)$, covering the $\mathrm{H}$ - and Heburning phases, and allowing for a moderate overshooting from convective cores $\left(\Lambda=l / H_{\mathrm{p}}=0.5\right)$. Rotation has not been included. Stellar tracks are computed under the assumption of constant-mass evolution. Additionally, for very massive 


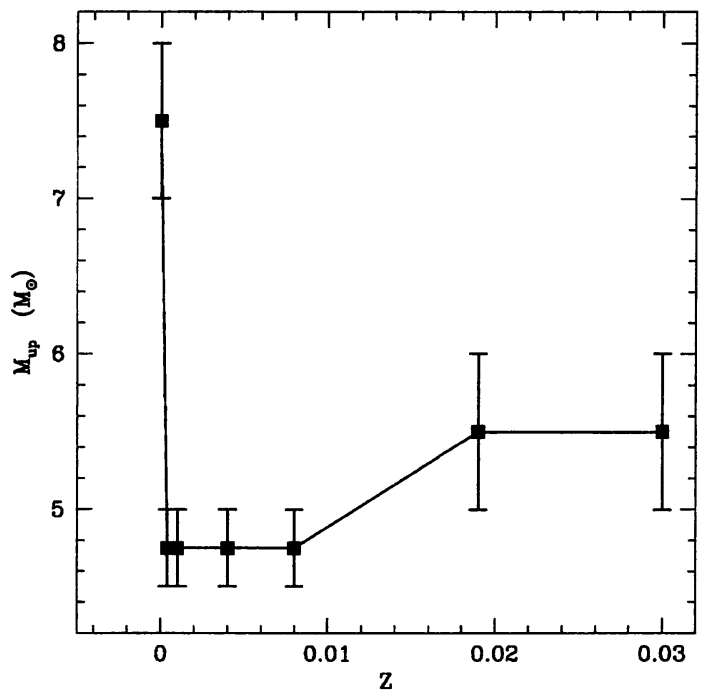

Figure 1. Expected behaviour of $M_{\mathrm{up}}$ as a function of metallicity. The prediction of this work for $Z=0$ is combined to the results of Girardi et al. (2000) for other metallicities.

models, with $M_{\mathrm{i}} \geq 120 \mathrm{M}_{\odot}$, we apply recent mass-loss rate $(\dot{M})$ prescriptions to account for: $(i)$ the radiation-driven winds at very low metallicities (Kudritzki 2002); and (ii) the intensification effect on $\dot{M}$ caused by stellar rotation (Maeder \& Meynet 2000).

\section{The critical mass $M_{\text {up }}$}

Let us first consider the critical mass $M_{\mathrm{up}}$, defined as the maximum initial mass for a star to develop an electron-degenerate C-O core, hence marking the boundary the limit between the class of low-intermediate-mass stars and that of massive stars. We find that it is around $\sim 7-8 \mathrm{M}_{\odot}$ for $Z=0$. Figure 1 displays the expected trend of $M_{\mathrm{up}}$ as a function of metallicity. It is essentially controlled by the mass of the He-core left at the end of the main sequence phase. At decreasing $Z$ and for CNO-dominated H-burning, the initial slight decline is due to the development of larger convective cores, because of larger luminosities and more concentrated energy sources. Then, the steeper rise at $Z=0$ reflects a reversed situation, i.e., smaller convective cores because of more extended burning regions. This is caused by the weaker temperature dependence energy generation rate when the p-p chain becomes competitive with the CNO-cycle, and this latter operates at higher temperatures as in the case of $Z \approx 0$.

\section{The onset of the triple- $\alpha$ reaction}

A peculiarity of $Z=0$ stars is that, differently from those with 'normal' chemical composition, the onset of the triple- $\alpha$ reaction can already happen during the $\mathrm{H}$ burning phase (see Marigo et al. 2001 for a detailed discussion). In few words, due to the lack of metals, initially the CNO-cycle cannot operate. The only 


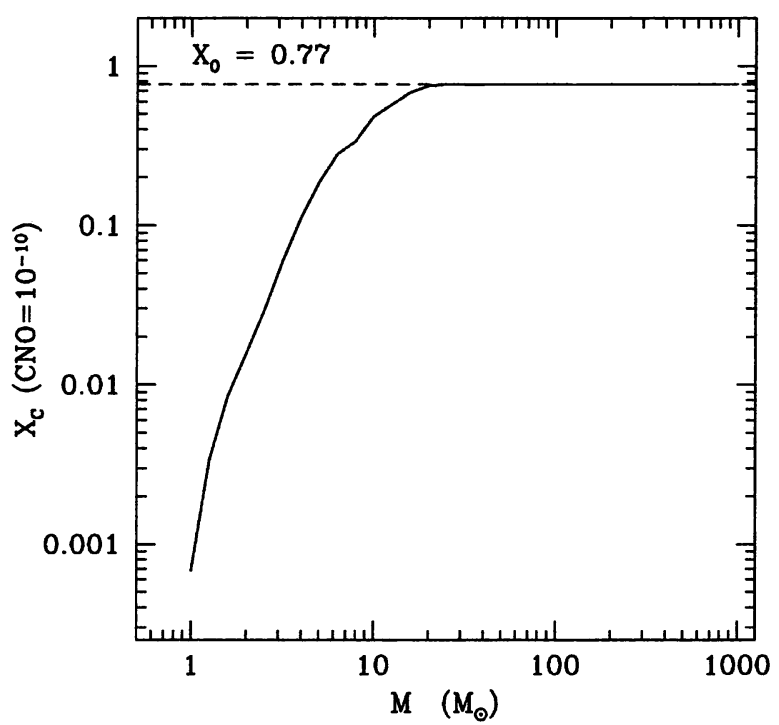

Figure 2. Evolutionary stage at the onset of the triple- $\alpha$ reaction, as a function of the initial mass $M_{\mathrm{i}}$. The ordinate reads the central hydrogen abundance when a CNO abundance of $10^{-10}$ (in mass fraction) is built up.

available energy sources are the gravitational contraction and the p-p chain. Due to the rather weak temperature-dependence of the latter, the central regions can contract until quite high temperatures are reached $\left(\sim 1-2 \times 10^{8} \mathrm{~K}\right)$, which lead to the onset of the $\alpha(2 \alpha, \gamma){ }^{12} \mathrm{C}$ reaction. As soon as a tiny abundance of ${ }^{12} \mathrm{C}$ is synthesised, then the CNO-cycle can be activated for the first time. As shown in Figure 2, such occurrence depends on the stellar mass, namely it takes place earlier and earlier at increasing $M_{\mathrm{i}}$. Specifically, in the high-mass domain the triple- $\alpha$ reaction ignites as soon as the star settles on the zero-age main sequence, before burning any significant amount of hydrogen.

\section{The location in the H-R diagram}

Figure 3 illustrates evolutionary tracks in the H-R diagram for selected values of the initial mass, under the assumption of constant-mass evolution. Based on these stellar tracks, a set of isochrones has been constructed (see Figure 4; available in electronic format at http://pleiadi.astro.it.

With respect to massive models $\left(M_{\mathrm{i}} \gtrsim 8 \mathrm{M}_{\odot}\right)$ we can make the following remarks: $(i)$ the H-burning phase is located at quite high effective temperatures such that, in combination with high luminosities, we expect these stars to emit large fluxes of UV photons (see Schaerer 2002); (ii) the He-burning phase takes place at decreasing effective temperatures at increasing stellar mass; (iii) stars with $8 \mathrm{M}_{\odot}<M_{\mathrm{i}}<70 \mathrm{M}_{\odot}$ remain always confined in the blue, and are not able to perform any redward excursion onto the Hayashi line before the ignition of central carbon. This will have important consequences for the expected surface chemical changes (see Section 6.). 


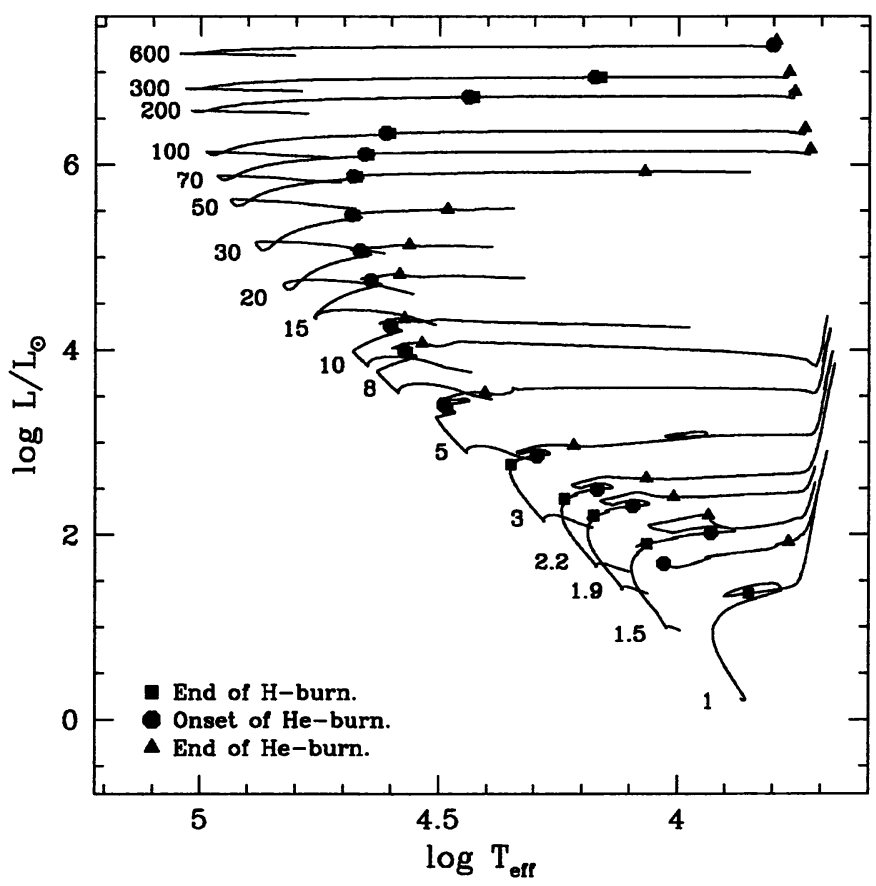

Figure 3. Evolutionary tracks in the H-R diagram for selected values of the initial stellar mass. Relevant stages are marked along the tracks.

\section{Surface chemical changes}

As we do not account for any rotationally-induced mixing, changes in the surface chemical composition are conditioned to the redward evolution of the star onto its Hayashi line (see Figure 3). Correspondingly, a deep convective envelope develops and extends into a chemically-variable profile (left by the receding convective core during the H-burning phase), so that newly-synthesised elements are dredge-up to the surface. Predictions for constant-mass evolution are shown in Figure 5. In summary, we find that the first dredge-up is experienced by stars with $0.7 \mathrm{M}_{\odot} \lesssim M_{\mathrm{i}} \lesssim 1.1 \mathrm{M}_{\odot}$, whereas the second dredge-up takes place in stars with $2.2 \mathrm{M}_{\odot} \lesssim M_{\mathrm{i}} \lesssim 8.0 \mathrm{M}_{\odot}$, and $M_{\mathrm{i}} \gtrsim 70 \mathrm{M}_{\odot}$. It should be noticed that further chemical changes may occur in the case of mass loss (see Section 7.).

\section{Mass loss and other evolutionary properties}

Mass loss is a crucial, but still uncertain, issue for primordial stellar evolution. We consider two possible driving-processes, radiative line acceleration and stellar rotation. To this aim, we adopt analytic formalisms recently presented by Kudritzki (2002), and Maeder \& Meynet (2000), respectively. In summary we find that, for a metallicity as low as $Z=10^{-4} Z_{\odot}$, radiation is not an efficient mass-loss mechanism, except for very massive stars with $M_{\mathrm{i}}>500 \mathrm{M}_{\odot}$.

Let us consider Figure 6, referring to a $250 \mathrm{M}_{\odot}$ model to which we apply Kudritzki's (2002) prescription. In this particular case, the amount of mass 


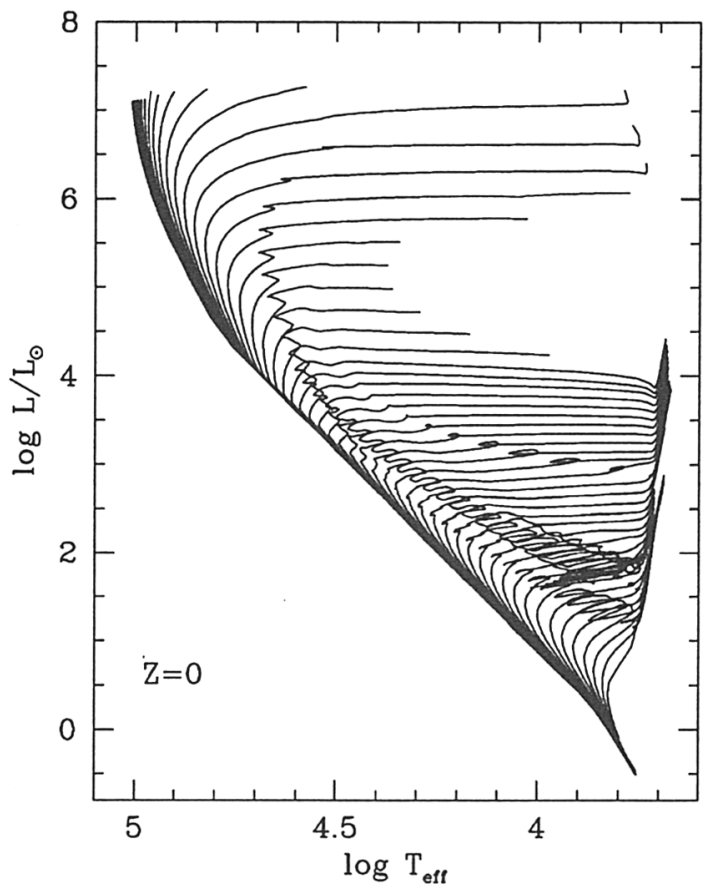

Figure 4. Theoretical isochrones in the HR diagram for the initial composition $(Z=0, Y=0.23)$. Ages span the range from $\log (t / \mathrm{yr})=5.0$ to 10.2 , at equally spaced intervals of $\Delta \log (t / y r)=0.1$. In all isochrones, the main sequence is complete down to a stellar mass of $0.7 \mathrm{M}_{\odot}$.
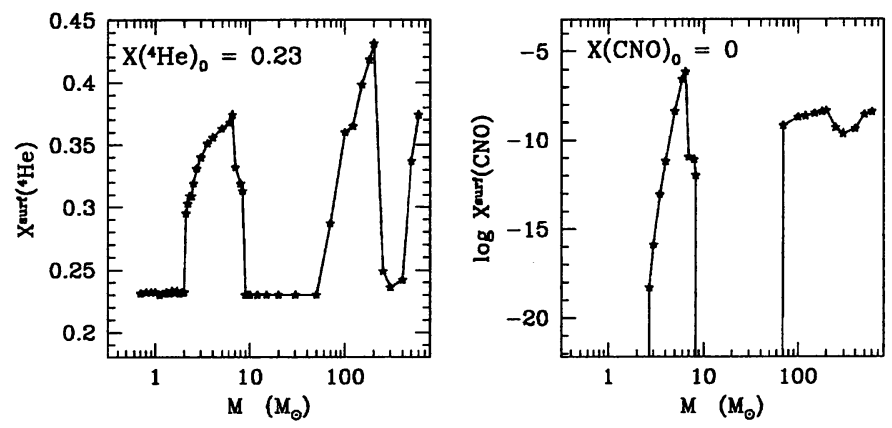

Figure 5. Surface abundances as a function of the stellar mass in the case of constant-mass evolution. The stage corresponds to the onset of the first thermal pulses on the AGB for low- and intermediate-mass stars, and the onset of carbon-burning for massive and very massive stars.

ejected during the evolution from the ZAMS to the onset of carbon-burning is negligible, i.e., $\sim 0.7 \mathrm{M}_{\odot}$. Note the large extension (in mass coordinate) of the convective cores during nuclear burnings, and the continuous transition from the end of core H-burning to the onset of core He-burning. In practice, we do not 


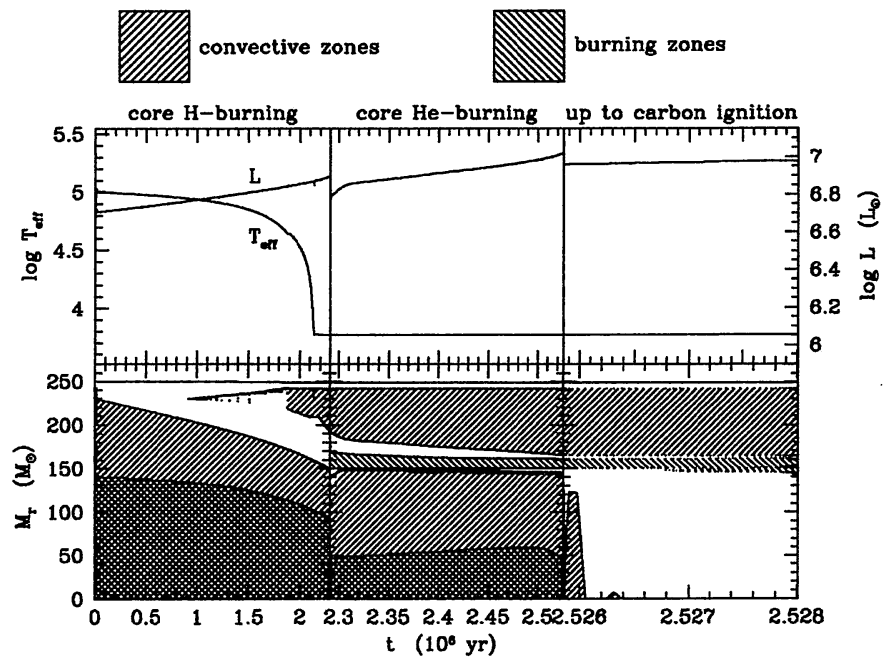

Figure 6. Evolutionary properties of the $250 \mathrm{M}_{\odot}$ model with the prescription for the purely radiative mass loss. Top panel: stellar luminosity and effective temperature as a function of time during the major nuclear burnings up to central carbon ignition. Bottom panel: convective and burning regions (in mass coordinate from the centre to the surface) across the stellar structure. The upper solid line represents the mass coordinate of the stellar surface.

expect any intermediate $\mathrm{H}$-shell burning phase in zero-metallicity (very) massive models, due to the already high temperatures reached in the central regions at the stage of exhaustion of central hydrogen.

Close to the end of the main sequence, the $250 \mathrm{M}_{\odot}$ model evolves towards lower effective temperatures until it approaches its Hayashi line. Consequently, an extended convective envelope develops so that the surface chemical composition is polluted with nuclear products of H-burning (mainly ${ }^{4} \mathrm{He}$ and ${ }^{14} \mathrm{~N}$ ).

Stellar rotation is expected to increase the purely-radiative mass-loss rates. As already discussed by Meynet \& Maeder (2001), the almost total preservation of the initial angular momentum may favour the attainment of the break-up velocity $(\Omega$-limit). In combination with the very large luminosities of very massive stars, the $\Omega \Gamma$-limit might also be approached. However, it should be noticed that (see Heger \& Langer 1998 for an extensive discussion), since a large fraction of the total angular momentum is deposited in the outermost layers, the ejection of a relatively small amount of mass from the surface should make the star to get rid of a significant part of its angular momentum, with consequent spinning down of the rotational velocity. As a consequence, the rotational effect on the mass-loss rate should also weaken.

The efficiency of stellar rotation in driving mass loss from zero-metallicity stars are examined in more detail by Marigo et al. (2003). This is an important issue for very massive stars, in relation to the possible occurrence of pairinstability supernovae (Heger \& Woosley 2002).

Acknowledgments. P.M. is grateful to N. Langer for providing his expertise on stellar rotation and helpful remarks on this work. 


\section{References}

Carr, B.J., Bond, J.R., Arnett, W.D. 1984, ApJ 277, 445

El Eid, M.F., Fricke, K.J., Ober, W.W. 1983, A\&A 119, 54

Girardi, L., Bressan, A., Bertelli, G., Chiosi, C. 2000, A\&A 361, 1023

Heger, A., Langer, N. 1998, A\&A 334, 210

Heger, A., Woosley, S.E. 2002, ApJ 567, 532

Kudritzki, R.-P. 2002, ApJ 577, 389

Maeder, A., Meynet, G. 2000, A\&A 361, 159

Marigo, P., Girardi, L, Chiosi, C., Wood, P.R. 2001, A\&A 371, 152

Marigo, P., Chiosi, C., Kudritzki, R.-P. 2003, A\&A 399, 617

Ober, W.W., El Eid, M.F., Fricke, K.J. 1983, A\&A 119, 61

Schaerer, D. 2002, A\&A 382, 28

Weiss, A., Abel, T. \& Hill, V. (eds.), 2000, The First Stars, Proc. MPA/ESO Workshop, ESO Astrophysics Symposia (Berlin: Springer)

\section{Discussion}

ConTI: I see that carbon is being created in this star and mixed to the surface. Is there sufficient carbon there to make any line driven stellar wind? In this star, could carbon drive a wind?

MARIGO: Indeed this is an interesting possibility, which we are going to investigate.

EL EID: If we are talking about chemical enrichment from primordial very massive objects you cannot exclude the effect of pair creation supernovae on this enrichment. Going back to 1983, we (El Eid et al. 1983; Ober et al. 1983) have shown that pair creation supernovae contribute significantly to the $\alpha$-elements, oxygen and nitrogen.

MARIGO: Yes, I agree with you. Actually, the role of pair-instability SNe in the early chemical enrichment should be investigated with the aid of observational tests on their nucleosyntetic signature (i.e., the predicted odd-even effect; see Heger and Woosley 2002), in comparison with that expected from SN II of lower progenitor masses.

HEGER: The $\mathrm{H}$ shell has a CNO mass fraction of $10^{-8}$. When products of central $\mathrm{He}$ burning are merged into this region, we always observed an almost explosive event in our calculations, since the CNO abundance, and thus the energy generation, are suddenly increased by several orders of magnitude, resulting in an almost 'explosive' event. Very likely, this situation is not treated appropriately in (our) 1-D code(s) with MLT convection treatment. (Similar results by other groups, e.g., by Woosley \& Weaver, remained unpublished.) Do you also find such events in your calculations? What is your opinion on what should happen here in detail? Remark on discussion of spin-down: see Heger \& Langer (1998).

MARIGO: As already discussed by Marigo et al. (2001), we actually find that, during the core He-burning phase, the growth of the convective core can reach the H-burning shell, so there some protons may be mixed down into the central regions (or, vice versa, some CNO may be injected into the shell.) A sudden flash of the H-burning nuclear energy generation is found to show up. I think this circumstance should be correctly handled only with a time-dependent mixing scheme. This is coupled with the nuclear network. In fact, convective and nuclear time scales may be comparable in such conditions, and the assumption adopted in our models of instantaneous mixing in convective regions may be no longer valid. 